\title{
Defect recognition by means of light and electron probe techniques for the characterization of me-Si wafers and solar cells
}

\author{
B. Moralejo, A. Tejero, V. Hortelano, O. Martínez, M.A. González, J. Jiménez \\ GdS-Optronlab, Dpto. Física Materia Condensada, Edificio I+D, Paseo de Belén, 1, 47011 Valladolid, \\ Spain
}

\begin{abstract}
Multicristalline Silicon (mc-Si) is the preferred material for current terrestrial photovoltaic applications. However, the high density of defects present in mc-Si deteriorates the material properties, in particular the minority carrier diffusion length. For this reason, a large effort to characterize the mc-Si material is demanded, aiming to visualize the defective areas and to quantify the type of defects, density and its origin. In this work, several complementary light and electron probe techniques are used for the analysis of both mc-Si wafers and solar cells. These techniques comprise both fast and whole-area detection techniques such as Photoluminiscence imaging, and highly spatially resolved time consuming techniques, such as light and electron beam induced current techniques and $\mu$ Raman spectroscopy. These techniques were applied to the characterization of different mc-Si wafers for solar cells, e.g. ribbon wafers, cast mc-Si as well as quasi-monocrystalline material, upgraded metallurgical mc-Si wafers, and finished solar cells.
\end{abstract}

\section{Introduction}

Nowadays, the photovoltaic industry is largely dominated by crystalline silicon, under the form of mono-crystalline (c-Si), or multi-crystalline (mc-Si). C-Si presents advantages, related to its low defect density and the optimized surface texture treatments. However, the elevated costs make preferential the use of mc-Si material grown from casting processes, in spite of its high concentration of intrinsic structural defects. Mc-Si is cheaper than $\mathrm{c}-\mathrm{Si}$, and is grown under the optimized squared wafer shape suitable for module production. The loss in efficiency in mc-Si with respect to c-Si is, therefore, compensated by its cost effectiveness.

The efficiency losses in mc-Si are mainly caused by the structural defects inherent to the growth procedure, e.g. grain boundaries (GBs), dislocations, and large incorporation of impurities [1-3]. These defects act as traps for the minority carriers, killing the minority carrier lifetime $(\tau)$, and shortening the carrier diffusion length ( $\mathrm{L}_{\mathrm{diff}}$ ), which negatively affects the energy conversion efficiency [2,3]. Some new approaches have appeared in recent years, trying to decrease the number of grains and GBs in $\mathrm{mc}-\mathrm{Si}$ [4], in particular, the quasi-mono $\mathrm{Si}$ (qm-Si) growth, in which the use of c-Si as seeds in a conventional casting furnace leads to large mono-crystalline areas, is attracting a great deal of attention [5]. Other approaches aiming to reduce the cost of the raw material run in parallel; in particular, the use of upgraded metallurgical grade silicon (UMG-Si), which faces the 
problems related to the high number of metallic impurities [6]. Other approaches, as for instance growing Si-Ribbons on a Sacrificial Template (RST), are also being considered; however, this method can introduce large thermal gradients leading to large number of defects [7].

An important effort is being carried out in recent years in the development of characterization tools permitting qualification and screening of the mc-Si wafers. Among them, both scanning and imaging techniques have been developed. In the scanning techniques the sample is locally excited by a focused laser beam, the corresponding signal is recorded, and associated with a couple of spatial coordinates $(\mathrm{x}, \mathrm{y})$, from which one can construct a 3-D map. The spatial resolution is determined by the size of the excitation probe, and the step size in the step by step motion of the sample. Among them, photoconductance decay (PCD), or quasi steady state photo-conductance (QSSPC) [8], microwave phase-shift ( $\mu \mathrm{W}$-PS) [9] and surface photovoltage (SPV) [10] techniques are time consuming and do not allow for high spatial resolution; while light beam induced current (LBIC) and electron beam induced current (EBIC) techniques [11,12], and microphotoluminescence $(\mu \mathrm{PL})$ [13], among others, are time consuming but allow for much higher spatial resolutions. Regarding imaging techniques, one can list infrared lifetime mapping (ILM) [14], carrier density imaging (CDI) [15], electroluminescence imaging (ELI) [16] and photoluminescence imaging (PLI) [17].

One of the most promising experimental tools for a fast qualification of mc-Si wafers is the PLI technique, because it permits, in a short time, a panoramic view of the main carrier capture centers in a full wafer. This technique is very promising for pure screening; though the profound understanding of the carrier trapping requires of techniques with high spatial resolution, and if possible with spectroscopic capabilities. Generally, LBIC and EBIC permit obtaining very high spatial resolutions, but present some important drawbacks; e.g. the very long time needed for a relatively large surface LBIC map (several hours), and the need for studying the wafers after surface treatments and electrode deposition, which does not permit to use them as screening methods in the early stages of the solar cell fabrication process. On the other hand, $\mu$ Raman spectroscopy is usually not employed for the characterization of large mc-Si areas, due to time consumption and experimental instabilities over long acquisition times. However, it can provide valuable information on the residual stress levels of the defect rich areas, because stress is relevant for defect gettering; therefore, one can try to correlate stress and electrical activity by combining Raman and LBIC, or microPL measurements [18].

We present in this work the characterization of multicrystalline Si wafers and cells obtained by different methods, by combining imaging techniques (PLI) with scanning optical and electrical techniques with high spatial resolution, such as $\mu$ Raman, LBIC and EBIC, thus taking the advantages of a quick inspection of the mc-Si wafers and solar cells and the detailed characterization of the electrically active defects. The study reveals the carrier trapping activity of different defects, showing that the highest charge trapping activity does not correspond to the GBs, but to intragrain defects (dislocations and subgrain boundaries), which play an active role as minority carrier lifetime killers. Silicon ribbons, traditional mc-Si as well as qm-Si and UMG-Si wafers and solar cells have been characterized. 


\section{Experimental}

Mc-Si wafers, using traditional raw material as well as UMG-Si, grown by conventional casting, qm-Si grown by using Cz-Si seeds [19], and solar cells fabricated from them following standard industrial methods, have been investigated. Also Si-ribbon wafers grown by the RST method [7] were characterized as grown, and after different annealing protocols aiming to relax the residual stresses.

For PLI, the whole wafers/solar cells were uniformly excited by means of four laser diodes $(\lambda=808 \mathrm{~nm}$, optical output power of $25 \mathrm{~W})$. The PL emission was collected with a Peltier cooled Si-CCD camera (PCO1300-Solar). Several filters were used to reject the laser light. A $12.5 \mathrm{~mm}$ focal length optical objective was coupled to the camera allowing the acquisition of the PL image from a whole wafer or cell. The exposure time was shorter than $2 \mathrm{~s}$ in the case of solar cells (acquired in reflection mode, using front-side illumination and front-side collection), being of the order of 1-2 min for obtaining the full wafer PLI (acquired in transmission mode, using front-side illumination and back-side collection). The LBIC apparatus was a home-made system operating with four excitation wavelengths supplied by two dual laser diodes (Omicron) with 639-830 nm and 853-975 nm emission lines, respectively. The laser beam enters into a trinocular microscope, which focuses it onto the sample surface. Excitation is typically made with low laser powers, in order to supply low injection levels, thus ensuring that the majority carrier density is not significantly affected. The excitation power, the back-reflected light power, the optical image (visualized by a Si-CCD camera), and the photocurrent signal are monitored simultaneously. A motorized $\mathrm{x}-\mathrm{y}$ stage allows for mapping the LBIC signals over regions of interest as large as $76.6 \times 114.5 \mathrm{~mm}^{2}$. Different microscope objectives were used (10X, $20 \mathrm{X}, 50 \mathrm{X}$ and 100X), which allow for both mapping large areas of the wafers, and also performing very high spatial resolution LBIC maps with the largest magnification objectives [20,21]. The EBIC measurements were carried out on a field emission scanning electron microscope (FESEM) (Carl Zeiss-LEO 1530) using beam energies between 10 and $30 \mathrm{keV}$. The electrical current generated by the electron beam is collected and amplified by a low noise current amplifier (MODEL DLPCA-200, FEMTO Messtechnik). $\mu$ Raman measurements were carried out at room temperature (RT) with a Labram UVHR 800 Raman micro-spectrometer from Horiba-Jobin Yvon. The excitation was done with a helium neon laser $(632 \mathrm{~nm})$ for which the penetration depth $(\approx 3 \mu \mathrm{m})$ was deep enough to minimize the influence of the surface on the Raman spectrum. The use of a precise spectral reference set up was mandatory because of the small Raman peak shift; a neon lamp located inside the optical entrance of the spectrometer, just ahead of the entrance slit, warranted a very stable spectral reference insensitive to the beam alignment. A LN2 cooled Si-CCD was used for detection.

\section{Results and Discussions}

The great advantage of the PLI technique for a quick inspection of the mc-Si wafers or solar cells, allowing for the fast identification of areas with trapping activity, is shown in 
Fig.1, where six PL images of solar cells fabricated from a qm-Si ingot, corresponding to several wafers from bottom to the top, are depicted. In this case, dark lines associated with the junction of four $\mathrm{c}-\mathrm{Si}$ seeds are appreciated, especially for the wafer corresponding to the bottom part of the ingot close to the seed (Fig. 1a). Some other defects, which seem to develop from this line, can be also observed, with a very large increase on their density as the position of the wafers moves from bottom to top, far from the seeds. The presence of these defects and their evolution from bottom to top should be related to the large thermal gradients appearing during the growth of the qm-Si brick; the strict control of the growth parameters appears essential for the obtention of bricks with large volumes free of defects.

In spite of the fast inspection provided by the PLI technique, its capability is somewhat limited by the spatial resolution, as mentioned. The lateral resolution is given by the size of the studied area and the pixel resolution; for instance, for the PL images shown in Fig.1, considering the standard solar cell dimensions $\left(156 \times 156 \mathrm{~mm}^{2}\right)$ and the number of pixels of the CCD $(1392 \times 1040)$, it is estimated at around $150 \mu \mathrm{m}$ per pixel. This lateral resolution is enhanced with respect to conventional point-by-point mapping techniques such as PCD, $\mu \mathrm{W}$-PS, etc., but is much lower than the resolution obtained by the electrical techniques LBIC and EBIC, and the $\mu \mathrm{PL} / \mu$ Raman mapping technique, which scale from a few micrometers down to the submicrometric range.

LBIC and EBIC techniques are thus suitable for a detailed inspection of the electrical activity of defects. For instance, Fig.2 shows LBIC maps obtained for a solar cell fabricated from conventional mc-Si. The simultaneous collection of the optical reflection image (obtained with a Si-CCD camera) of the analyzed area in our LBIC set-up allows to reveal the grains with different orientations, since each grain disperses the light in a different manner. Most of the detected dark lines correspond to intragrain defects, showing that the GBs are not the main trapping active defects in mc-Si, but the main sites for carrier trapping, and therefore for killing the carrier lifetime, are defects inside the grains. This result is highlighted when increasing the LBIC spatial resolution; the resolution was enhanced by increasing the optical magnification of the objective as well as decreasing the scanning step size. In particular, Fig. 2e-f show the LBIC map obtained with very large lateral resolution (magnification $100 \mathrm{X}, 3 \mu \mathrm{m}$ step size). The vertical dark line in Fig. 2e is clearly related to an intragrain defect. Fig. 3 shows also LBIC maps with increased resolution for a solar cell fabricated from UMG-Si. In this case, extended defect areas surrounded by clouds of tiny dark spots are observed in the high resolution LBIC maps; these tiny spots are likely related to metallic impurity precipitates decorating the extended defects.

An important additional advantage of LBIC is that it allows for an easily quantification

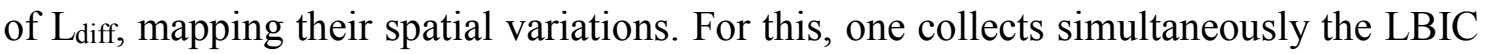
and reflected light maps at least for two wavelengths, which permits the calculation of both the external and the internal quantum efficiencies (EQE/IQE) [20]. Fig. 4 shows the LBIC $(a, d)$, optical reflection $(b, e)$ and IQE $(c, f)$ maps obtained by exciting a conventional mc-Si sample with 639 and $830 \mathrm{~nm}$ laser lines. The analyzed area shows two grains (see the optical images) and two dark lines associated with intragrain defects. The IQE values take into account the correction by the reflectivity of the sample surface, which, as 
previously mentioned, is highly dependent on the orientation of each individual grain; therefore, it can introduce changes in the electrical response that can hinder the LBIC contrast if one does not take care of it. The IQE values have been evaluated by monitoring both the incident and reflected light power on the sample surface by the use of two

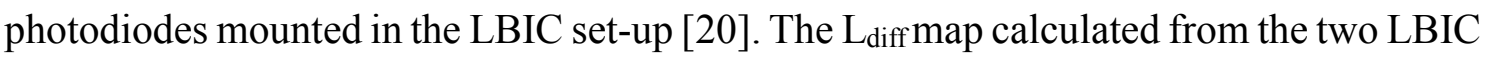
measurements is shown in Fig. $4 \mathrm{~g}$. The decrease of $\mathrm{L}_{\text {diff }}$ around the GBs is almost negligible, while around the two electrically active dark lines it is severely depressed, close to $80 \%$. These results must be regarded with care for a reliable analysis; a precise calibration of the set-up is needed. In particular, the reflected light collected by the objective presents a large dependence with its numerical aperture (NA), being very important for the reliable estimation of the IQE map [20]. In this sense, the calculation of $\mathrm{L}_{\text {diff }}$ would be more realistic when using large NA objectives, which are more efficient for the collection of both specular and diffusive reflected light than low magnification objectives with low NA, which mainly collect the specular reflected light.

EBIC inspection of conventional mc-Si samples shows quite similar results than those obtained by LBIC, with somewhat higher spatial resolution, see Fig.5. The SEM images (Fig.5a, c and e) allow also to distinguishing the different grains, through the differences in their surface textures. The EBIC map of Fig.5b, at low magnification, shows a small trapping activity of the GBs, and a much higher trapping activity of the intragrain defects. Fig.5d corresponds to the EBIC map of the square area marked on Fig.5b, but at higher magnification. The corresponding SEM image (Fig. 5e) shows the surface texture of the area selected for the study, which corresponds to only one grain. The dark line, related to an intragrain defect, shows a marked inhomogeneity, without correlation to the surface features revealed by SEM. This result remarks the non homogeneous trapping activity along the dark line, because of the non homogeneous decoration with impurities.

LBIC and EBIC are thus high spatial resolution characterization techniques. In the detrimental side, both are time consuming and need for specific material preparation. A good compromise for mc-Si analysis would consist of a first quick inspection of the wafers/solar cells by means of PLI. This will permit the localization of the trapping areas; a further analysis of local areas using high spatial resolution techniques would provide a plus of understanding about the trapping features. A good correlation between the PL images and the LBIC maps is demonstrated in Fig. 6a, which shows a LBIC map of an area of a solar cell fabricated from qm-Si, previously investigated by PLI (Fig.1). This LBIC map was obtained with low spatial resolution (small magnification and large step sizes), which does not require long acquisition time. In spite of this, one can observe a contrast higher than obtained by PLI. This is due to the high sensitivity to charge capture of the electrical techniques (both LBIC and EBIC) and the improved signal to noise ratio with respect to the PLI apparatus used in our experiments, which is limited by the spectral range of the detector. Fig.6b shows LBIC maps of the same area obtained with increased resolution.

The combination of the electrical characterization provided by LBIC (or EBIC) with the optical characterization provided by $\mu$ Raman is also interesting in view of establishing a 
relation between stress and electrical activity. For instance, Fig. 7 shows the electrical characterization by means of LBIC of a portion of a RST Si-ribbon wafer. In this case, a metallization process was necessary in order to fabricate a Schottky junction to collect the carriers. The comparison of the optical image with the LBIC map reveals the existence of defects aligned parallel to the pulling direction of the ribbon, which correspond mainly to GBs (see the arrows in Fig. 7). Some other structural defects such as twins, also observed in the optical image, do not show significant electrical activity. In order to investigate the residual strain in the ribbon wafers, $\mu$ Raman maps were carried out around a GB, as shown in Fig. 8. The Raman results clearly show a strain distribution around the GB line, which is associated with the large thermal gradients of the RST process. Later on, the wafer was annealed and the Raman measurements were repeated, showing a decrease of the residual stress.

Finally, the same complementary characterization was performed for conventional mc-Si samples, as shown in Fig. 9. In this case, a $\mathrm{KOH}$ etching procedure allowed to reveal the extended defects, allowing to differentiate GBs and dislocations lines (DL), see Fig. 9a. The Raman maps obtained in this area show high residual strain associated only with the DL, Fig. 9b. LBIC investigations after metallization of this sample showed again no electrical activity associated with the GB, but only the DL gave a dark contrast. These results evidence the role of the residual stress around the DL on its electrical activity, which can be likely explained in terms of the impurity gettering around the DL under the strain field. The difference with the GB character might be explained in terms of the stress relieve around the GBs as compared to the residual stress remaining around the intragrain defects.

\section{Conclusion}

The trapping activity of defects in mc-Si associated with GBs and intragrain regions have been evaluated by means of the fast inspection by PLI and by high spatial resolution optical and electrical techniques, $\mu$ Raman, LBIC and EBIC. The PLI technique is very suitable for wafer screening, while the other techniques appear as powerful tools for the study of the trapping mechanisms in mc-Si. In particular, the LBIC set-up is very well suited to carry out analysis of large areas as well as to obtain very high spatial resolution, by the combination of different magnification objectives and sample motion step sizes. Electrically active defects seem to present residual stress, while stress relaxed defects did not present electrical activity.

\section{Acknowledgement}

This work was supported by the Research Projects VA166A11-2 ("Consejería de Educación, Junta de Castilla y León” - Spain), IPT-420000-2010-022 (INNPACTO program, "Ministerio de Ciencia e Innovación", Spain) and ENE2014- 56069-C4-4-R (Ministerio de Economía y Competitividad, Spain). 


\section{References}

[1] K. Yang, G.H. Schwuttke, and T.F. Ciszek, J. Cryst. Growth 50 (1980) 27

[2] S. Pizzini, A. Sandrinelli, M. Beghi, D. Narducci, F. Allegretti, and S. Torchio, J. Electrochem. Soc. 135 (1988) 155

[3] M. Rinio, S. Peters, M. Werner, A. Lawerenz, and H.J. Möller, Solid State Phenom. 82-84 (2002) 701

[4] H. Zhang, L. Zheng, X. Ma, B. Zhao, C. Wang, and F. Xu, Journal of Crystal Growth 318 (2011) 283

[5] X. Gu, X. Yu, K. Guo, L. Chen, D. Wang, and D. Yang, Sol. Energy Mater. Sol. Cells $101(2012) 95$

[6] S. De Wolf, J. Szlufcik, Y. Delannoy, I. Perichaud, C. Habler, and R. Einhaus, Sol. Energy Mater. Sol. Cells 72 (2002) 49

[7] F. De Moro, A. Focsa, K. Derbouz, A. Slaoui, N. Auriac, H. Lignier, and P.Keller, Phys. Stat. Solidi c 9 (2012) 2092

[8] R.A. Sinton, and A. Cuevas A, Appl. Phys.Lett. 69 (1996) 2510

[9] O. Palais, L. Clerc, A. Arcari, M. Stemmer, and S. Martinuzzi, Mat. Science Eng. B 102 (2003) 184

[10] J. Lagowski, P. Edelmant, M. Dexter, and W. Henley, Semicond. Sci. Technol. 7 (1992) A185

[11] F.J. Vorster, and E.E. van Dyk, Rev. Sci. Instrum. 78 (2007) 013904

[12] O. Breitenstein, J. Bauer, M. Kittler, T. Arguirov, and W. Seifert, Scanning 30 (2008) 331

[13] H. Sugimoto, M. Inoue, M. Tajima, A. Ogura, and Y. Ohshita, Jpn. J. Appl. Phys. 45 (2006) L641

[14] M. Bail, J. Kentsch, R. Brendel, and M. Schulz, Proceedings of the 28th IEEE-PVSC, Anchorage, AK, p. 99 (2000)

[15] J. Isenberg, S. Riepe, S.W. Glunz, and W. Warta, J. Appl. Phys. 93 (2003) 4268

[16] T. Fuyuki, H. Kondo, T. Yamazaki, Y. Takahashi, and Y. Uraoka, Appl. Phys. Lett. 86 (2005) 262108

[17] T. Trupke, R.A. Bardos, M.C. Schubert, and W. Warta, Appl. Phys. Lett. 89 (2006) 044107

[18] G. Sarau, S. Christiansen, M. Holla, and W. Seifert, Sol. Energy Mater. Sol. Cells 95 (2011) 2264

[19] J. Barredo, V. Parra, I. Guerrero, A. Fraile, and L. Hermanns, Prog. Photovolt: Res. Appl. 22 (2014) 1204

[20] B. Moralejo, M.A. González, J. Jiménez, V. Parra, O. Martínez, J. Gutiérrez, and O. Charro, J. Electron. Mater. 39 (2010) 663

[21] B. Moralejo, V. Hortelano, M.A. González, O. Martínez, J. Jiménez, S. PonceAlcántara, and V. Parra, Phys. Status Solidi C 8 (2011) 1330 

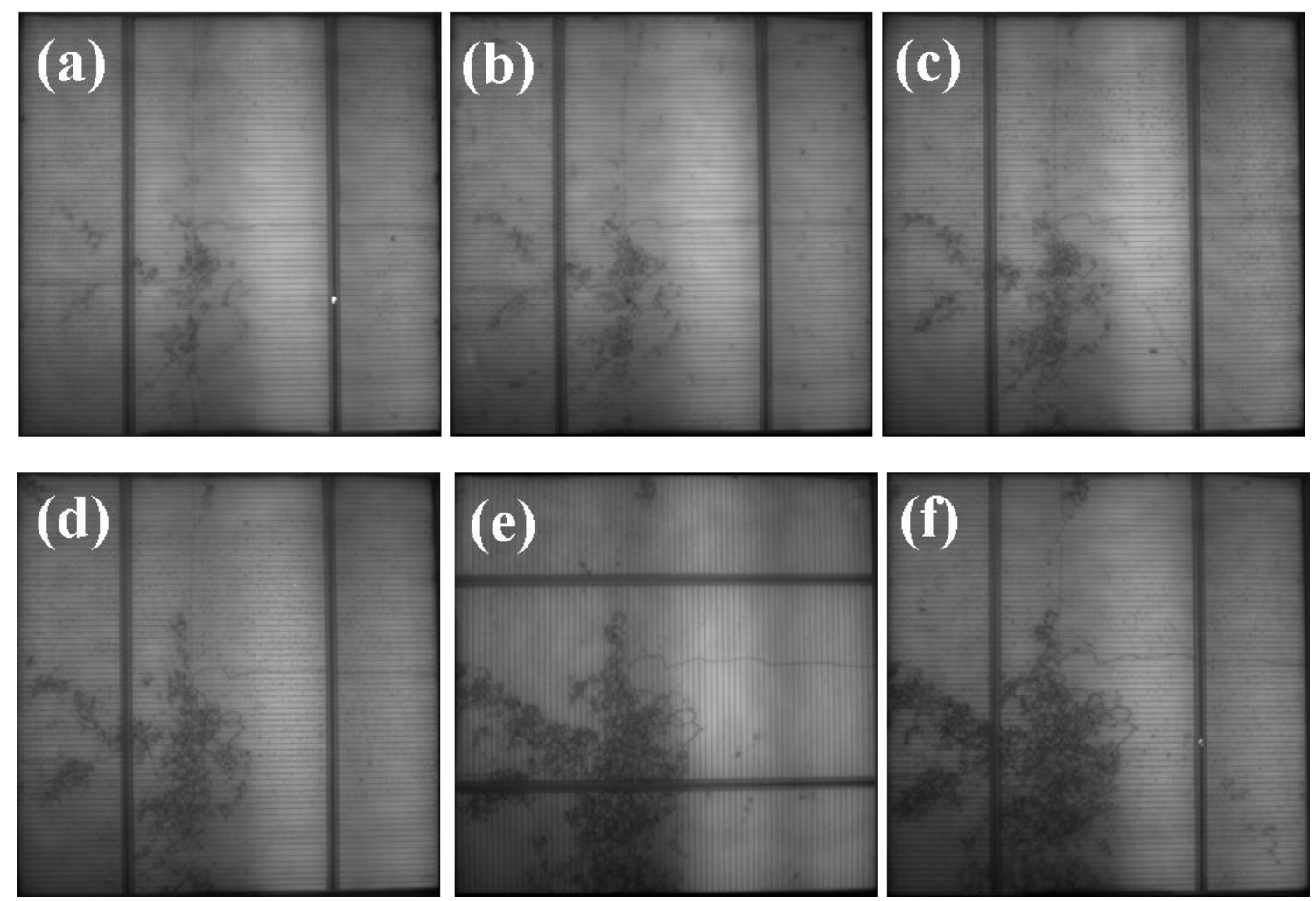

Figure 1. PLI results of solar cells grown from a qm-Si ingot. The position of the wafers varies from bottom (a) to top (f) of the ingot. 

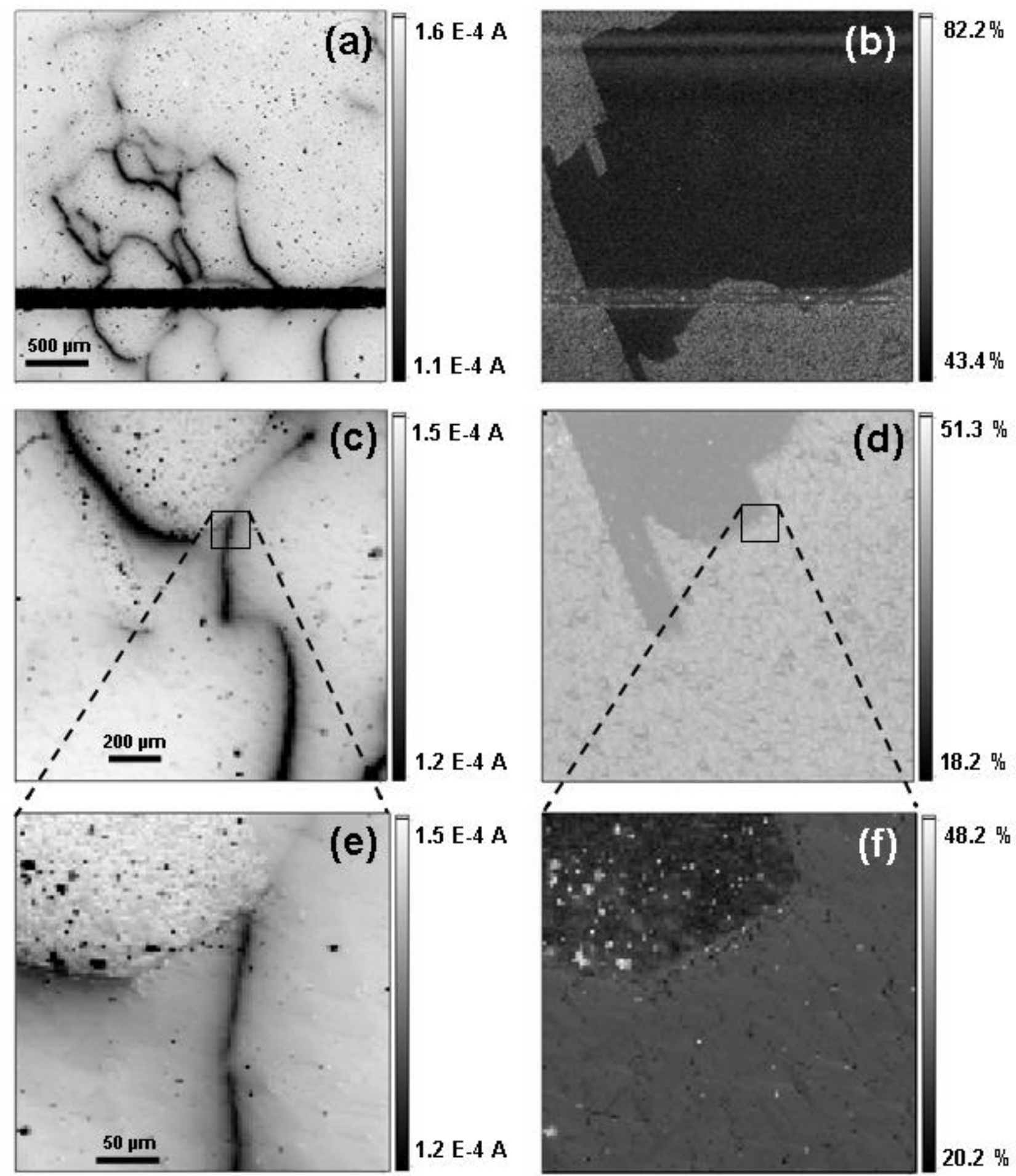

Figure 2. LBIC maps (a, c, e) and optical reflection images (b, d, f) of a conventional mc-Si solar cell, obtained with increasing resolution. $(\mathrm{a}, \mathrm{b})$ : objective $=20 \mathrm{X}$, step size $=$ $15 \mu \mathrm{m} ;(\mathrm{c}, \mathrm{d})$ : objective $=20 \mathrm{X}$, step size $=10 \mu \mathrm{m} ;(\mathrm{e}, \mathrm{f}):$ objective $=100 \mathrm{X}$, step size $=3$ $\mu \mathrm{m}$. 


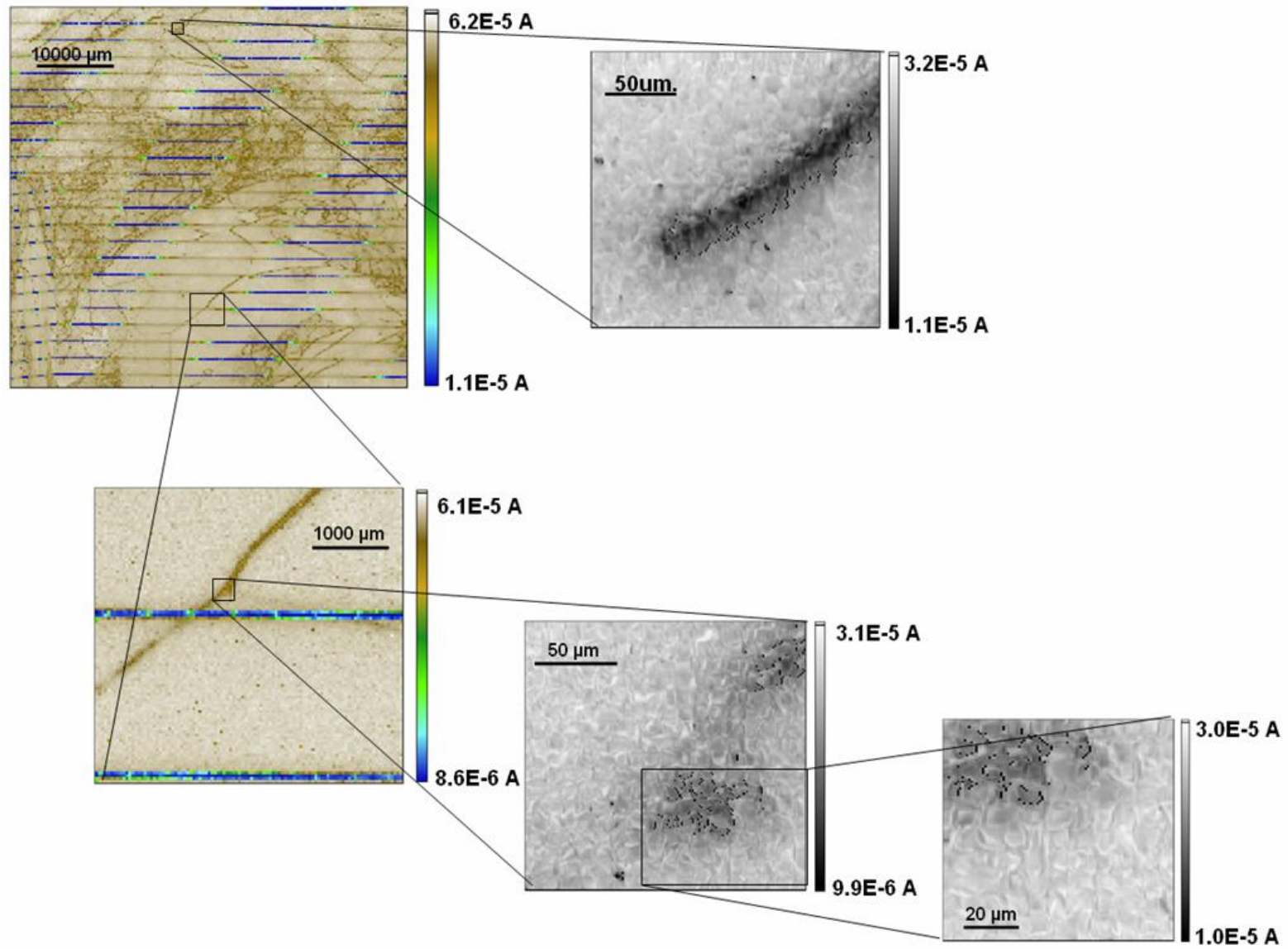

Figure 3. LBIC maps with different spatial resolutions of a UMG-Si solar cell. 

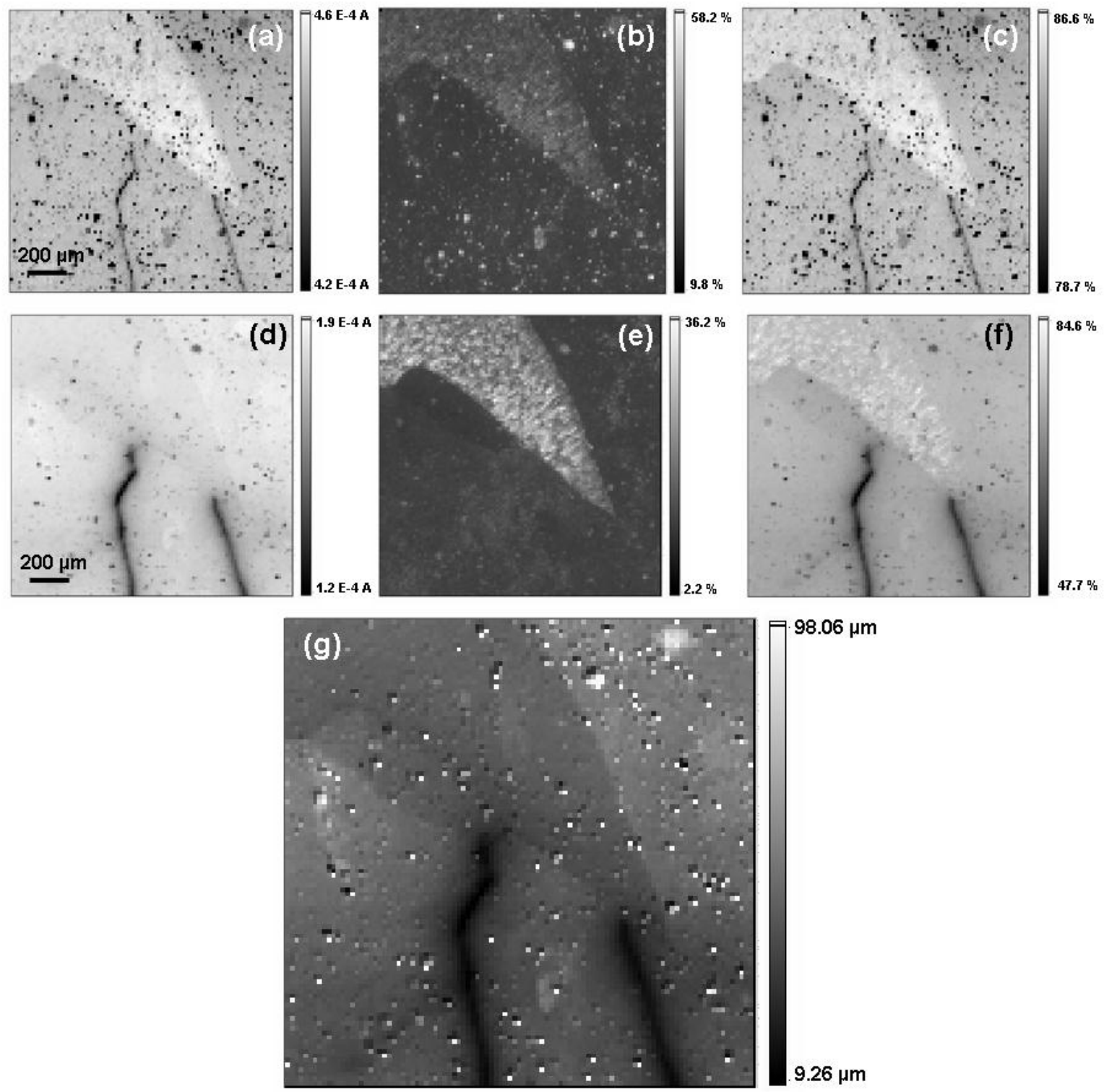

Figure 4. LBIC maps (a, d), optical reflection images (b, e) and IQE maps (c, f) of a portion of a solar cell obtained at $639 \mathrm{~nm}(\mathrm{a}, \mathrm{b}, \mathrm{c})$ and $830 \mathrm{~nm}(\mathrm{~d}, \mathrm{e}, \mathrm{f})$. (Objective $=20 \mathrm{X}$, step size $=14 \mu \mathrm{m}) ; \mathrm{g}) \mathrm{L}_{\text {diff }}$ map calculated from the two IQE maps. 

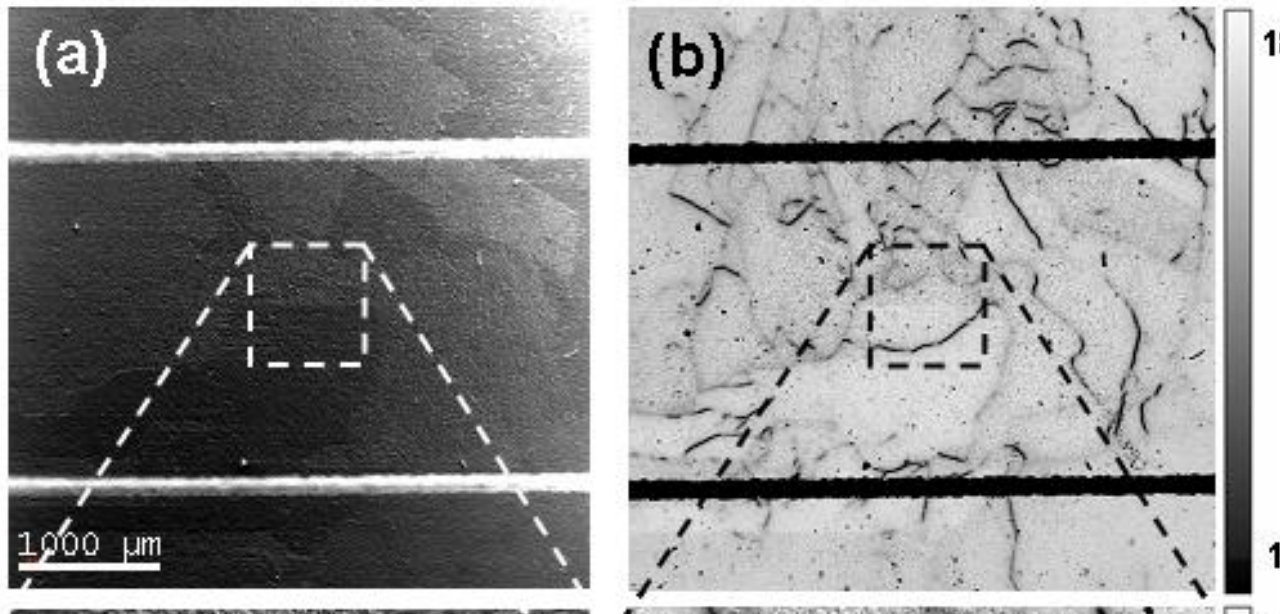

18500 (a.u.)
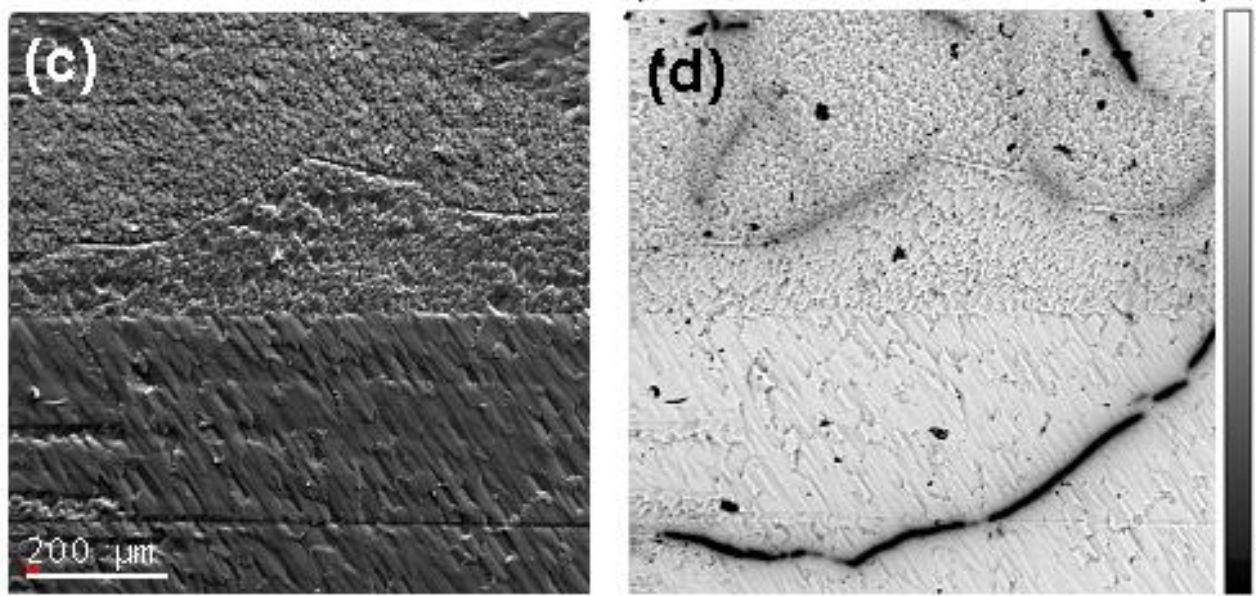

17000 (a.u.)

18500 (a.u.)
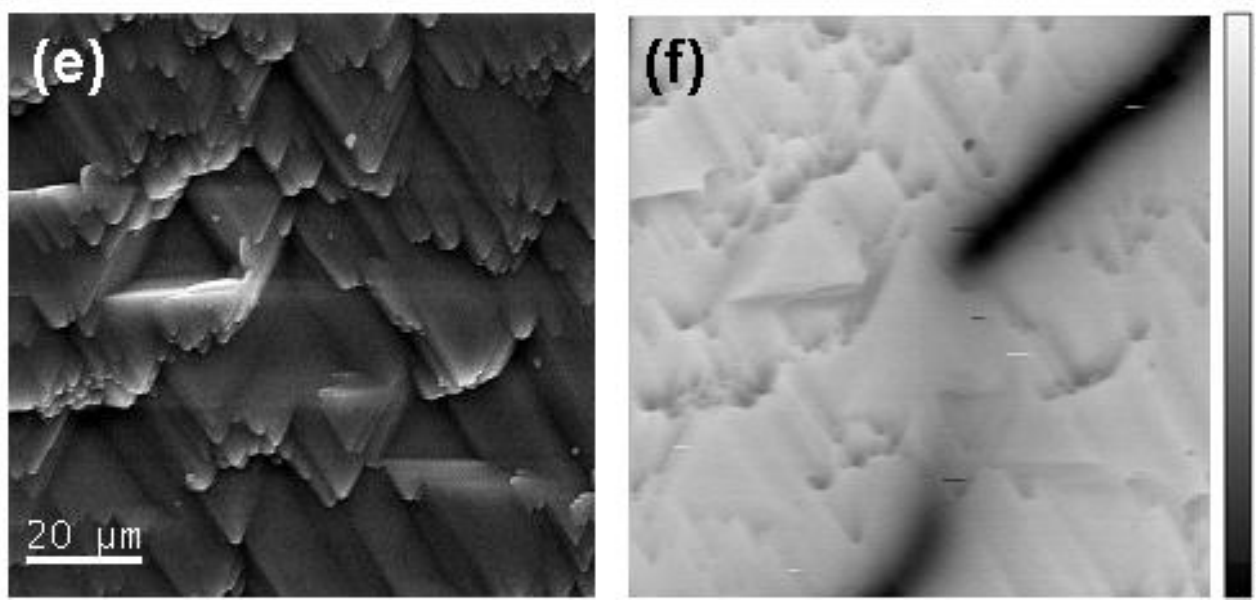

17000 (a.u.)

7900 (a.u.)

7700 (a.u.)

Figure 5. SEM (a, c, e) and EBIC (b, d, f) images obtained with increasing resolution. $\left(E_{b}=30 \mathrm{keV}\right)$ on a conventional mc-Si sample. 

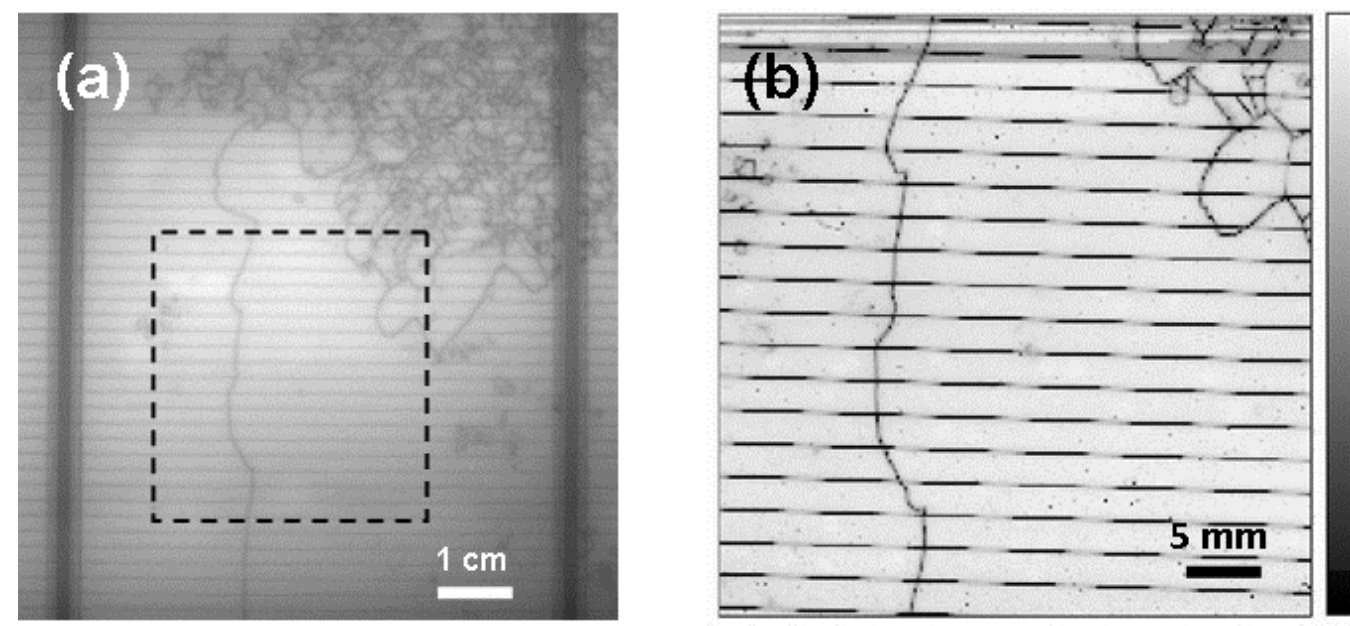

1.7 E-3 A

$2.2 \times 10^{-3} \mathrm{~A}$

$2.1 \times 10^{-3} \mathrm{~A}$

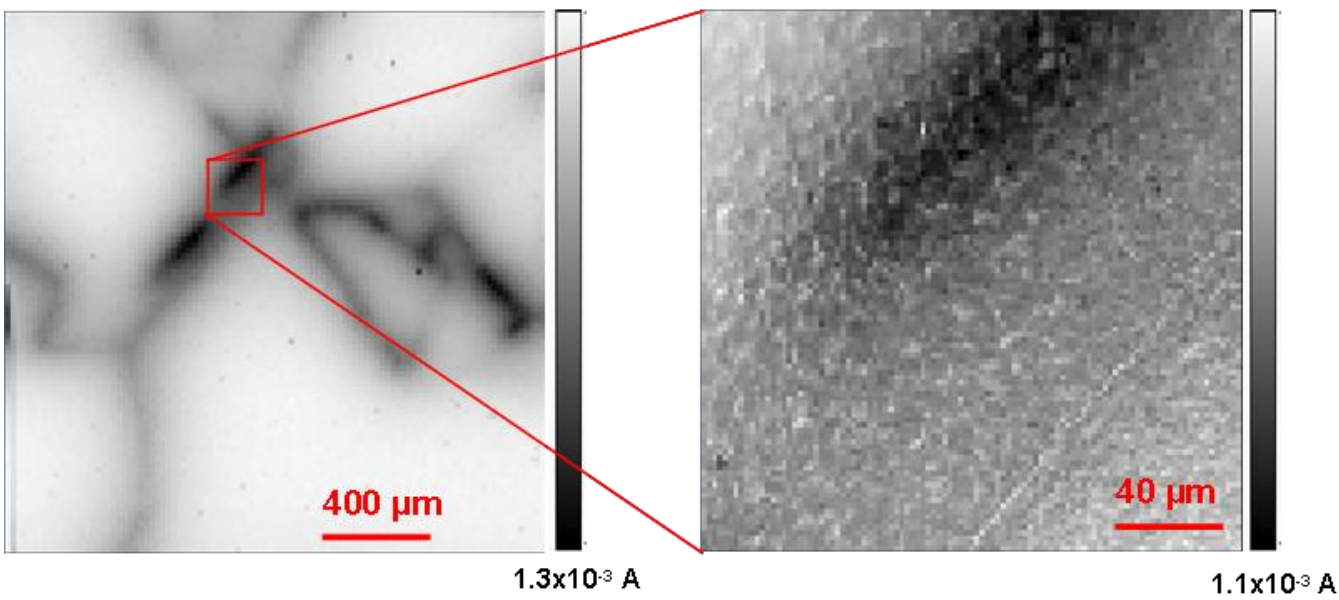

Figure 6. a) PL image of a portion of a solar cell fabricated from a qm-Si wafer and b) LBIC map of the squared white frame area shown in a). $\left(\lambda_{\text {exc }}=853 \mathrm{~nm}\right.$, objective $=20 \mathrm{X}$, step size $=200 \mu \mathrm{m}$ ); c) LBIC maps obtained with increased resolution of the observed defective lines. 


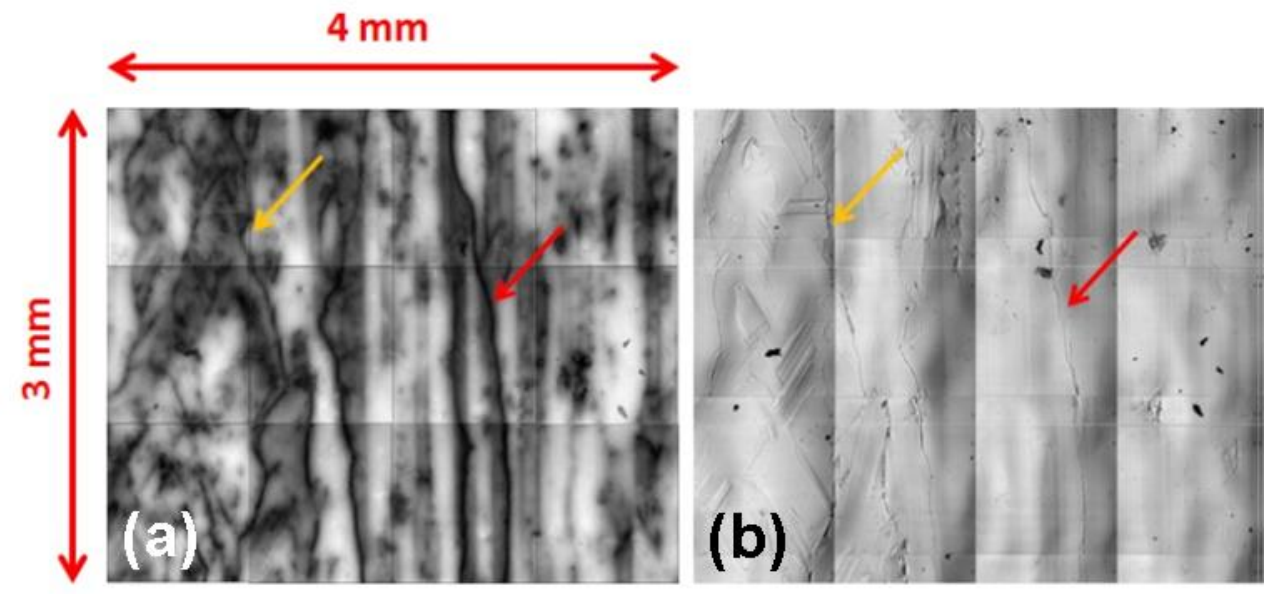

Figure 7. LBIC (a) and optical image (b) of a portion of a Si-ribbon grown by the RST process (the arrows indicate electrically active GBs). 


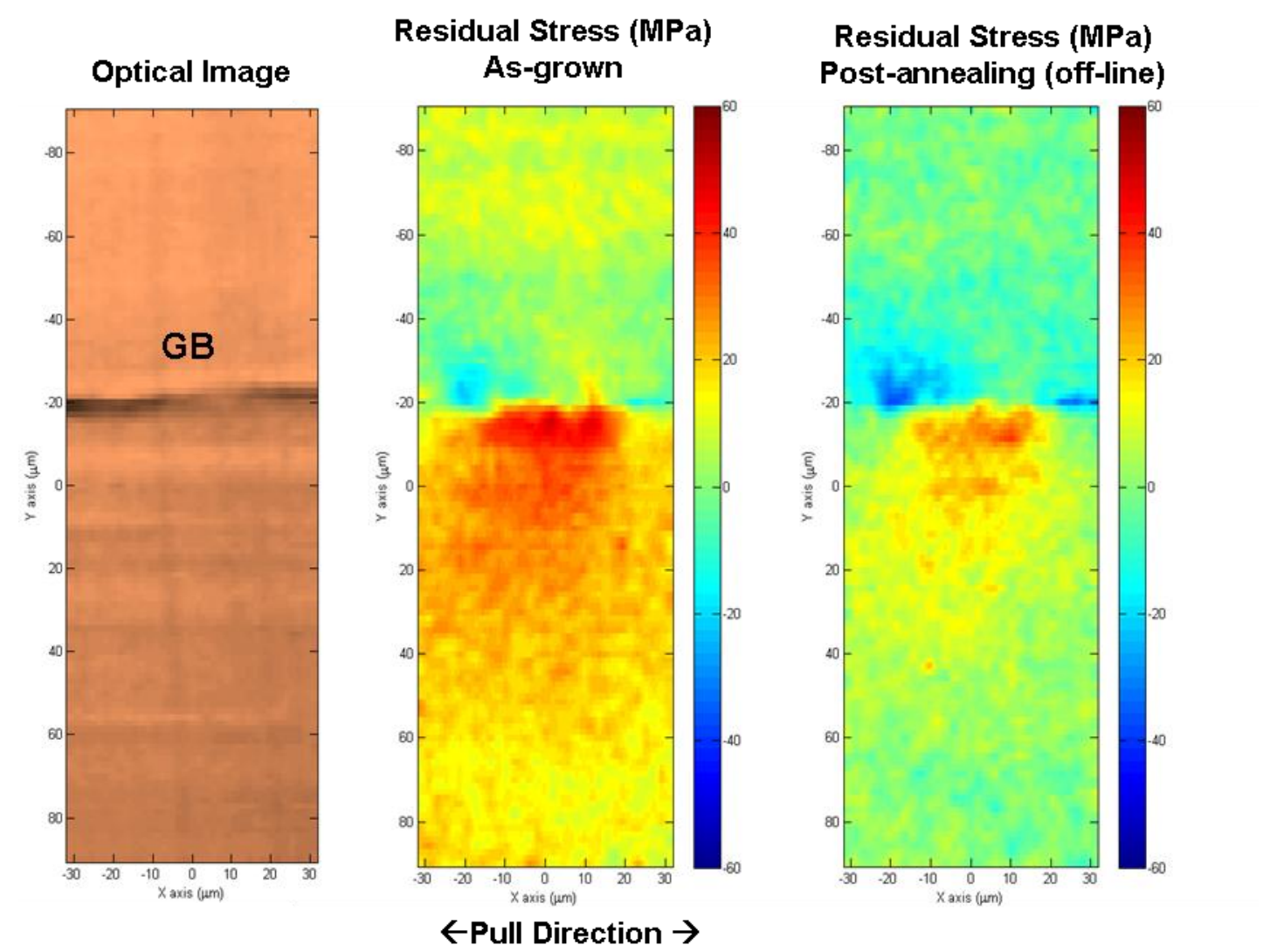

Figure 8. Optical image of a portion of a Si-ribbon, showing a GB (a), and residual Raman strain maps of this area for the as-grown sample (b) and the same sample after annealing (c). 

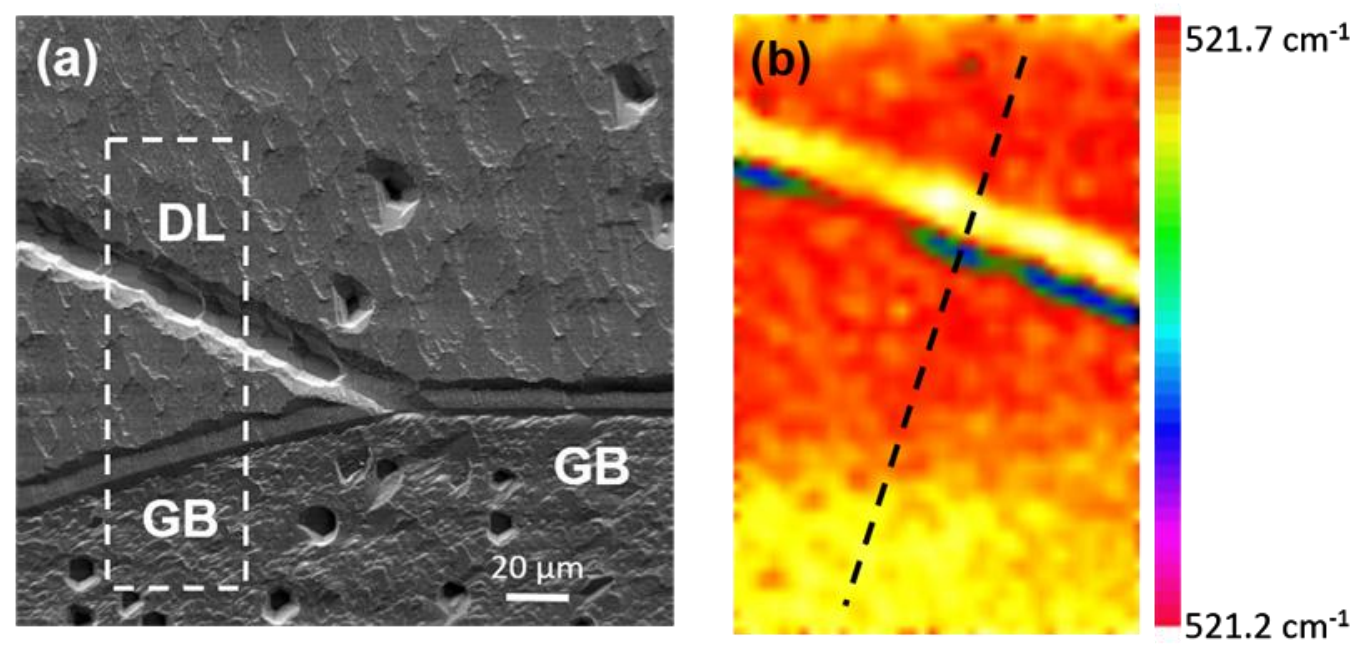

Figure 9. (a) SEM image of a conventional mc-Si wafer, etched by $\mathrm{KOH}$, showing a GB and a DL and (b) Raman map of the squared region shown in (a). 